\title{
APLICAÇÕES E LIMITAÇÕES DA MODELAGEM EM AGRICULTURA - REVISÃO
}

\author{
Simone Toni Ruiz Corrêa ${ }^{1}$, Rogério Lorençoni ${ }^{1}$, Durval Dourado Neto ${ }^{1}$, Fábio Vale Scarpare ${ }^{2}$, \\ Rafael Vivian ${ }^{3}$, Eduardo Toni Ruiz ${ }^{4}$ \\ ${ }^{1}$ Depto. de Produção Vegetal, Escola Superior de Agricultura 'Luiz de Queiroz', ESALQ/USP, Piracicaba - SP. Fone: (019) \\ 3429-4458, struiz@esalq.usp.br; \\ ${ }^{2}$ Depto. de Engenharia de Sistemas Agrícolas - ESALQ/USP, Piracicaba - SP. \\ ${ }^{3}$ Embrapa Meio-Norte, Teresina-PI. \\ ${ }^{4}$ UNESP - Instituto de Biociências, Rio Claro-SP.
}

\section{RESUMO}

Modelos podem ser usados para investigar uma série de assuntos relacionados à produção vegetal, seja para facilitar o entendimento quanto ao comportamento da cultura dentro de seu contexto ambiental, explorar seu potencial produtivo sob certas condições, verificar hipóteses, melhorar o conhecimento de processos, estimular a integração interdisciplinar, predizer o comportamento de um sistema ou ser utilizado como ferramenta de gerência e tomada decisória. Como vantagens, lhes são atribuídos o custo menos oneroso do que experimentos convencionais que, cada vez mais, possuem um elevado custo de instalação, manutenção e obtenção dos dados sem, no entanto ter a pretensão de substituí-los -, a velocidade na obtenção de resultados, o uso em diferentes escalas e a criação de cenários alternativos. Suas limitações abrangem a dificuldade na validação de modelos já existentes, alto custo de obtenção de alguns dados, como por exemplo, séries meteorológicas, variabilidade espacial e seleção de dados de entrada. Muitas são as formas de avaliar o desempenho de um modelo e, por vezes, deduções errôneas são tiradas, descaracterizando a utilização desta ferramenta. O uso da modelagem em agricultura ainda é bastante incipiente no Brasil, quando comparado a outros países. Este estudo tem como objetivo abordar alguns dos principais conceitos e mecanismos de modelagem em agricultura, com o intuito de contribuir e estimular as pesquisas, ainda escassas nessa área, e sua difusão.

Palavras-chave: modelagem, agricultura, linguagem computacional, análise sensitiva

\section{APLICATIONS AND LIMITATIONS OF CROP MODELS - BRIEF REVIEW}

\section{ABSTRACT}

Models can be used to investigate a series of issues related to crop production, facilitating the knowledge regarding crop behavior in its environmental context, to exploit its potential production under certain conditions, testing hypotheses, improve the knowledge of the process, stimulate interdisciplinary integration, diagnosis of a system behavior or it can be used as a management tool, supporting decisions. As advantages, the costs are cheaper than conventional trials, which have a high cost of installation, maintenance and acquisition of data - not intended to replace them - the speed is fast in obtaining results, it is used at different knowledge scales and the creation of alternative scenarios. Its limitations include the difficulty in validating existing models, the high cost of obtaining some data, for instance, historical meteorological information, spatial variability and selection of input data, for example. There are many ways to evaluate the performance of a model and sometimes erroneous deductions are made, misunderstanding the use 
of this tool. The use of crop modeling is still incipient in Brazil compared to the other countries. This study aimed at addressing some key concepts and mechanisms in crop modeling, in order to contribute and stimulate the research, still scarce in this area, and its diffusion.

Key words: modeling, agriculture, computational language, sensitive analysis

\section{INTRODUÇÃO}

\section{Aspectos gerais e principais aplicações da modelagem em agricultura}

O uso de modelos na agricultura é datado desde os primeiros cultivos na história da humanidade. Naquele tempo era sabido que ao plantar determinada cultura em determinada época e modo, seu desenvolvimento ocorreria de maneira que a colheita seria em outra época. Esse modelo mental foi sendo aprimorado pelos agricultores com o passar dos tempos, baseado nas suas próprias experiências. A utilização de modelos objetivando quantificar os efeitos das variáveis ambientais no crescimento e desenvolvimento das culturas vem ocorrendo há mais de 270 anos. Quando Réaumur, em 1735 , fez a associação entre temperatura e o desenvolvimento de culturas, ele estava propondo um dos modelos empíricos mais eficientes que se conhece em agrometeorologia, baseado no acúmulo de graus-dia. A partir da década de 50, trabalhos mais elaborados vieram formalizar o que seria o alicerce da modelagem em agricultura (Zadocks \& Rabbinge, 1985), onde um dos trabalhos mais relevantes foi o desenvolvido pelo Professor C. T. de Wit, da Universidade de Wageningen - Holanda, em 1958, intitulado "Transpiration and crop yield", seguido em 1968 pelo clássico trabalho "Photosynthesis of leaf canopies". Após tais publicações, observou-se um crescente interesse pela área de modelagem em diversos países do mundo, inclusive no Brasil.
Os diferentes modelos de culturas agrícolas, com maior ou menor nível de empirismo em função do conhecimento sobre os fenômenos estudados, normalmente consistem no balanço do carbono - que depende da interceptação e da utilização da luz - e de um balanço hídrico - que depende da demanda atmosférica e da utilização da água pelas plantas. Estes processos fisiológicos podem ser associados à absorção dos nutrientes e às perdas causadas por pragas, doenças e plantas daninhas. A agregação destes conhecimentos através de equações matemáticas resulta em modelos que podem simular o crescimento e a produção das culturas (Penning de Vries, 1982).

Em condições favoráveis ao crescimento, os processos fisiológicos e o rendimento 'potencial' das culturas são determinados principalmente pelas características varietais e por variáveis climáticas, como temperatura, fotoperíodo e radiação. Esse fato torna importante a análise do crescimento e desenvolvimento da cultura em diferentes situações, pois significa que o potencial de rendimento das culturas difere entre locais e anos, e entre épocas no mesmo local (Kropff et al., 1995; Pereira et al., 2002). A quantificação do potencial produtivo em diferentes áreas indica aquelas mais apropriadas para a produção de determinada cultura, além de servir também para estimativa da produtividade 'real', considerando eventuais quebras de rendimentos (Pedro Jr. et al., 1983; Marin et al., 2000). Este raciocínio é a base do método da Zona Agroecológica (MZA), ou modelo de Doorenbos e Kassam 
(1994).

O crescimento e desenvolvimento das plantas dependem fundamentalmente dos processos de fotossíntese e respiração. A pesquisa da fotossíntese é refletida em sofisticados modelos, os quais existem para predizer o crescimento das plantas, dados da elevação solar, geometria das folhas, penetração da luz, taxa individual da fotossíntese, etc. No entanto, a maioria dos recursos e esforços tem sido despendida no estudo da fotossíntese, sendo a respiração considerada apenas um processo de perda de carbono (Pereira \& Machado, 1987). Coube a McCree (1970) e Thornley (1970), mostrar que esses dois processos são interligados e interdependentes, e que a respiração faz parte efetiva do processo de utilização e distribuição dos carboidratos fotossintetizados.

Um dos primeiros modelos propostos para a estimativa da produção de fotoassimilados em função da área foliar e ângulo de inserção das folhas, além de considerar outros elementos inerentes ao dossel da cultura, como velocidade do vento, $\mathrm{CO}_{2}$, radiação e transpiração, foi o SPAM, no qual posteriormente foi incluída uma equação considerando a atividade estomática (Duncan, 1975). O modelo ELCROS foi desenvolvido para simular o crescimento da planta de milho (Wit \& Goudriaan, 1978), assim como o modelo SIMAIZ (Duncan, 1975). Entretanto, todos são limitados, pois não levam em consideração todos os fatores e interações entre o ambiente e a planta, caso do modelo SIMAIZ, que considera que a produção de fotoassimilados seja função única das interações entre radiação, temperatura e área foliar.

Mais tarde, Andrade et al.(1991) propuseram que o rendimento da cultura é expresso pela interação da radiação incidente com a eficiência de interceptação da radiação incidente, a eficiência de conversão da radiação interceptada e a partição de fotoassimilados. Nesse caso, a radiação incidente pode aumentar segundo a latitude; entretanto, deve-se considerar a taxa de desenvolvimento por unidade de tempo térmico, uma vez que temperatura afeta o ciclo da planta (Dourado Neto, 1999).

Diversos autores desenvolveram modelos que simulam o desenvolvimento da planta, o acúmulo de matéria seca nos seus diferentes órgãos e o índice de área foliar, em função de parâmetros fenológico e climatológico (Keulen et al., 1982; Keulen \& Wolf, 1986; Spitters et al., 1986).

Em geral, estes modelos podem ser usados para investigar uma série de assuntos relacionados à produção vegetal. Eles servem para facilitar o entendimento quanto ao comportamento da cultura dentro de seu contexto ambiental, podendo ser usados em escalas regional e global, para prever ou explorar o potencial produtivo sob certas condições (Ittersum et al., 2003; Bouman et al, 1996), sendo, muitas vezes, utilizados como ferramenta de gerência e tomada decisória (Meinke \& Hochman, 2000). Além disso, os modelos de simulação são utilizados para verificar teorias e testar hipóteses, melhorar o conhecimento sobre um determinado processo (Munakata, 1995; Boote et al., 1996), além de estimular uma integração interdisciplinar e descrever, de forma concisa, um grande número de observações isoladas. Por sua vez, estes modelos podem predizer o comportamento do sistema, em combinações e condições não experimentadas anteriormente. De maneira geral, a aplicações destes modelos é bastante ampla, e incluem desde zoneamento agroecológico a estudos sobre o efeito das mudanças ambientais ou sócio-econômicas na agricultura (BoumaN et al., 1996).

No Brasil, apesar de alguns exemplos de sucesso, as técnicas de modelagem não estão sendo desenvolvidas, nem utilizadas em seu pleno potencial. Desenvolvimento e utilização de modelos são conceitos distintos. Para se formar massa crítica em modelagem e aproveitar todo o benefício 
didático e científico dos modelos é necessário que os mesmos sejam desenvolvidos, ou se já existem modelos similares em outros países, que sejam adaptados, levando-se em conta as condições brasileiras. Exemplos de adaptações de modelos desenvolvidos no exterior e adaptados às condições do Brasil podem ser consultados em Corrêa (2008) e Lorençoni et al. (2010).

A construção de modelos simplificados é interessante para torná-los mais acessíveis ao público não especializado. Tais modelos podem ser feitos extraindo-se todos os detalhes excessivos do modelo complexo, usando-se análises de sensibilidade e pela seleção da variável principal do sistema. Diferentes modelos simplificados podem ser construídos a partir de um mesmo modelo complexo, sendo que a diferença entre eles irá depender dos diferentes objetivos de sua aplicação. Um exemplo do acima exposto: nos anos 90, Spitters argumentou que um modelo de simulação de produtividade de cultura chamado SUCROS (Simple and Universal CROp growth Simulator), no qual a abordagem da fotossíntese era incluída, poderia ser simplificado, incorporando apenas os processos determinantes que mais afetam o crescimento das culturas. Esta foi a primeira abordagem de um modelo que, mais tarde, seria batizado como LINTUL (Light INTerception and UtiLization) (Bouman et al., 1996). Em Corrêa (2008), a rotina do modelo LINTUL-soja, originalmente na linguagem FORTRAN ('FORmula TRANslation", muito usada nas décadas de 80 e 90 principalmente na Europa, porém pouco difundida no Brasil), foi replicada para a linguagem Visual Basic desenvolvida pela Microsoft e popularmente difundida no país. Dessa maneira, o modelo simplificado LINTUL pode ser usado por um maior número de usuários (difusão do conhecimento).
No atual cenário, os módulos de simulação dos processos componentes parecem ser aqueles que mais rapidamente podem ser incorporados e utilizados, como por exemplo, os que simulam o balanço hídrico para orientação do manejo da irrigação.

\section{Modelos e Modelagem}

Diversos pesquisadores das mais variadas áreas desenvolvem seus estudos e acumulam conhecimento e informações passíveis de serem ampliadas - é a necessidade inerente do homem em compreender como a natureza e seus componentes funcionam. Por sua vez, para que os resultados dessas pesquisas sejam confiáveis, existe a necessidade da realização de experimentos - que envolve custo de instalação, manutenção e obtenção dos dados - além do tempo necessário para a condução dos mesmos. Em muitos casos, não existe a possibilidade de instalação de experimentos, exigindo-se que a tomada decisória seja rápida. Para contornar situações como estas, propõem-se a 'descrição de um sistema real', na escala do objetivo do estudo e com a interpretação dos fenômenos envolvidos correlacionada ao nível de exatidão obtida na sua descrição. Para que esta 'simplificação' seja possível, exige-se que exista um elevado conhecimento dos conceitos básicos de funcionamento do sistema em questão.

Sendo assim, conceitua-se como modelagem a ferramenta científica baseada na representação simplificada de um sistema que, na maioria dos casos, pode ser composta por um conjunto de símbolos e relações matemáticas logicamente ordenadas (modelos). Alguns protocolos computacionais empregam apenas 'regras' que modelam o sistema investigado, como por exemplo, modelos variacionais fuzzy (em 
inglês, incertos), autômatos celulares, algoritmos genéticos e redes neurais.

Basicamente, a modelagem tem como objetivos: (i) estabelecer 'diagnósticos de funcionamento' de um ou mais processos; (ii) identificar 'carências científicas' a serem exploradas. O uso de modelos permite obter um conhecimento integrado das suas partes em diferentes escalas espaciais e temporais estabelecendo diretrizes e definindo hipóteses assumidas sobre o sistema real.

Há diversas classificações propostas para diferenciar os modelos. Uma delas os divide em matemáticos e de simulação.

$\mathrm{O}$ primeiro caso refere-se a representações matemáticas de um fenômeno, podendo ser de três tipos: (i) empíricos; (ii) mecanísticos e (iii) estocásticos (Pautian et al., 1992). Os modelos de simulação englobam um ou mais modelos matemáticos, representando fenômenos mais complexos.

Modelos empíricos são aqueles que se baseiam puramente em dados observados, ou seja, na interação quantitativa entre os elementos considerados no mesmo. Não há muito interesse em explicar detalhadamente porque o sistema responde de tal forma. Nesse tipo de modelo utilizam-se dados empíricos, variáveis ou constantes consagradas que fazem parte do conhecimento de determinada ciência. Quando o interesse é descrever primariamente a resposta de um sistema de forma geral, o método empírico pode ser utilizado satisfatoriamente. Normalmente, estes modelos fundamentam-se em relações derivadas a partir de análise de regressões e, quando gerado de uma série de dados longa, podem ser suficientemente exatos para serem usados em dadas situações. No entanto, devem ser usados com ressalva para extrapolações, em condições similares àquelas em que foram gerados. Apesar da ressalva de extrapolação sem ajustes para diferentes condições daquelas de sua origem, segundo Dourado Neto et al. (1998a), um modelo obtido por análise de regressão, quando baseado no entendimento de processos de desenvolvimento de um sistema, por exemplo, pode ser mais valioso que um complexo modelo mecanístico, baseado somente no conhecimento de suas respostas.

Modelos mecanísticos são aqueles que têm sua estrutura baseada na descrição do processo que ocorre no sistema real considerado, ou seja, existe a tentativa de se considerar os princípios físicos, químicos e biológicos que ocorrem no sistema, tentando entender o sistema em termos dos mecanismos presentes. Tais modelos apresentam grandes dificuldades na obtenção dos dados necessários para o seu desenvolvimento; por outro lado, apresentam pouca restrição à extrapolação geográfica e espacial dos resultados, quando comparados ao modelo anterior. São os mais versáteis dentre os tipos de modelos matemáticos (Pautian et al., 1992).

O modelo é estocástico quando baseado em uma ou mais variáveis de natureza randômica (Law \& Kelton, 1991), ou seja, se o estado do sistema for determinado pelo momento anterior, afetado por uma probabilidade devida ao acaso. São modelos em que o fator 'probabilidade' está presente (Thornley, 1976).

Os modelos de simulação permitem fazer simulações de longo prazo, sendo realizadas geralmente a um baixo custo. Conforme Addiscott (1993), eles podem ser divididos em: (i) determinísticos, em que um conjunto de eventos leva a um resultado único e definido e (ii) estocásticos, em que a incerteza é considerada na sua estrutura, ou seja, apresentam algum grau de probabilidade associado à sua resposta, característica comum dos modelos empíricos. Uma das características mais importantes da simulação é sua habilidade de modelar comportamentos ou variações randômicas (tempo de processos e taxas, por exemplo). Dizer que uma variável de um modelo é randômica não significa dizer que 
ela é indefinida nem sequer imprevisível mas, sim, que o fenômeno modelado tende a variar estatisticamente, sendo probabilisticamente previsível. Portanto, modelos que não possuem natureza randômica - os determinísticos - possuem comportamento determinado, uma vez que os dados de entrada sejam definidos. Modelos de simulação determinísticos podem ser obtidos da mesma maneira que modelos estocásticos. A diferença básica está no método de análise a ser adotado para cada um dos modelos. Nos determinísticos, o resultado de uma única simulação fornece uma medida exata da performance do modelo. Para os estocásticos, várias interações são necessárias para que se possa obter um resultado médio que gere uma estimativa confiável da performance do modelo.

Para as situações onde as incertezas exercem pouca influência no resultado final, a aplicação de modelos determinísticos pode ser cabível e de boa aceitação, como por exemplo, na adubação de solos 'homogêneos', ou no dimensionamento de sistemas de irrigação. Porém, quando as variáveis de interesse podem apresentar-se extremamente variáveis quanto a seus valores como, por exemplo, a temperatura média de determinada localidade, o procedimento estocástico vem a contemplar as incertezas, fazendo possível que não só um único valor seja admitido como resposta final, mas sim, várias possibilidades - cada uma associada à determinada probabilidade de ocorrência. Tais procedimentos são comumente aplicados via cadeia de Markov, análises espaço-temporais, e método de Monte Carlo.

$\mathrm{Na}$ realidade, a maioria dos modelos de simulação é uma mistura de empiricismo e mecanicidade. Mesmo os mais mecanísticos dos modelos usam empiricismo em algum nível hierárquico de sua estrutura (Boote et al., 1996).
Para entender o comportamento de determinado nível do sistema, muitas vezes é necessário compreender como este é influenciado pelo comportamento de um nível abaixo. Assim, um sistema aparentemente complexo pode ser desdobrado em níveis relativamente mais simples (Jones, 1986). Quando o conhecimento de determinado nível explicativo é suficientemente extensivo, tendo sido o modelo deste sistema criado com base nesse sólido conhecimento, pode não ser mais necessário testar esses modelos comparando-os com a realidade (Wit, 1974).

Deve ser ressaltado, entretanto, que todos os modelos são abstrações. $\mathrm{O}$ grau de abstração permitido é um valor de um julgamento a ser feito no contexto dos objetivos. A chave para uma efetiva modelagem está no equilíbrio entre o realismo e a abstração na reprodução do sistema (Santos, 1986). Não existe, portanto, um modelo certo ou errado, mas sim modelos com diversos graus de aplicação para diversas circunstâncias. Não existe também um modelo universal que forneça uma solução para todos os problemas. Contudo, os modelos devem continuar a ser construídos e adaptados às diversas situações particulares. Os profissionais que trabalham com essas ferramentas devem definir claramente seus objetivos antes de pensar em construí-los, enquanto que os usuários devem escolher aqueles que melhor resolvam suas necessidades específicas (Thornley \& Johnson,1990; Dourado Neto et al., 1998a).

\section{Componentes do processo de modelagem e a avaliação do modelo}

Sumariamente, 'modelo matemático' é definido um conjunto de relações matemáticas que descrevem as mudanças nas variáveis de estado como resultado dos diferentes processos que ocorrem nesse 
sistema (Wit, 1978; Jones et al., 1987). Sistema, por sua vez, é a coleção de componentes e suas relações que são agrupadas para estudar uma parte do mundo real. Esta seleção depende dos objetivos do estudo e é uma visão simplificada da realidade.

Entende-se como variável de estado as 'quantidades' que descrevem as condições dos componentes no sistema, e podem mudar com o tempo à medida que os componentes do sistema interagem com o meio. Se as variáveis de estado mudam no tempo, os modelos são dinâmicos. Como exemplo de variáveis de estado, pode-se citar a quantidade total de biomassa de determinada cultura. É importante se ressaltar que as variáveis de estado são, em grande parte dos modelos de simulação de crescimento e desenvolvimento de culturas, a característica de interesse do modelador (Wit, 1978; Jones et al., 1987).

Entradas (Inputs) são os fatores do meio ambiente que influenciam o comportamento do sistema, mas que não são influenciados pelo mesmo, como por exemplo, as variáveis meteorológicas. Inputs também se referem a variáveis moduladoras, que agem sobre os fluxos e variáveis de estado. Se um sistema não tem inputs, ele não é afetado pelo ambiente e é denominado um sistema fechado. Inputs podem variar com o tempo. Saídas (Outputs) são os resultados do modelo que são de interesse do modelador.

Os parâmetros são características dos componentes do modelo que geralmente são constantes através do tempo simulado. A distinção entre parâmetros e inputs não é sempre clara. Geralmente, inputs são diretamente dependentes do tempo, enquanto parâmetros são constantes ou dependem do estado do sistema, mas não necessariamente do tempo. Por exemplo, os parâmetros podem definir a resposta funcional da fotossíntese à luz, a resistência do solo à densidade de fluxo de água, a resposta funcional da variação temporal do índice de área foliar e a perda de água pela planta através do processo evapotranspiratório. (Jones et al., 1987).

As interrelações entre os componentes e o sistema, e algumas vezes entre variáveis de estado no sistema, ocorrem como resultado de vários processos. Por exemplo, a biomassa de uma cultura (variável de estado) muda como resultado dos processos de fotossíntese e respiração; o conteúdo resultado da chuva ou da evapotranspiração e drenagem.

Fluxos, por sua vez, representam as entradas e saídas dos compartimentos das variáveis de estado. Os fluxos, que algumas vezes são os próprios processos, são sempre expressos na unidade de tempo.

Há muitas ferramentas disponíveis para facilitar a aplicação de modelos e simulações de sistemas contínuos. Em particular, os símbolos têm sido definidos para expressar em diagramas os componentes e os processos dos sistemas (inputs, outputs, variáveis de estado, limites do sistema e inter relações entre eles, por exemplo). Esses procedimentos são úteis para desenvolver um modelo matemático do sistema. Além disso, tais diagramas são fundamentais para o profissional comunicar a essência do modelo com outras pessoas.

$\mathrm{O}$ procedimento de modelagem é iniciado por intermédio do entendimento correto dos objetivos do modelo a ser construído, que devem ser claros e bem definidos, baseados no conhecimento do problema a ser resolvido. Caso eles sejam amplos, devem ser subdivididos em ordem de prioridade. Os objetivos devem ser suficientemente completos, para que a partir deles, possam ser determinados os parâmetros, inputs, o conjunto de equações a ser utilizada, a metodologia aplicada e as características dos resultados que serão obtidos.

No processo dinâmico de construção do programa computacional, em que as 
equações são transformadas em linguagem computacional - programas de computadores especiais desenvolvidas para apresentar as funções de rotina que são características da maioria dos estudos de simulação -, é aconselhável que os resultados dos cálculos apresentados pelo computador sejam comparados com outros resultados já existentes na literatura, para evitar que os erros não sejam encontrados, devido à complexidade e da magnitude do programa.

Os modelos devem ser avaliados de acordo com os objetivos inicialmente propostos. Há várias maneiras de se avaliar o desempenho de um modelo. Uma delas é a análise sensitiva, ou de sensibilidade, que consiste em avaliar os parâmetros ou entradas do modelo, considerando sua importância relativa para os resultados da simulação. Esta análise também fornece um mecanismo para testar a simulação nos extremos, e usando os valores extremos dos parâmetros vão rigorosamente testar o modelo em termos de estabilidade e lógica matemática. A análise de sensibilidade se inicia elegendo-se as principais saídas (resultados, outputs) do modelo, e se estabelece uma condição normal do modelo, com as melhores estimativas de cada parâmetro e entradas. Tomam-se, então, os parâmetros ou entradas a serem avaliadas quanto ao seu efeito no modelo (sensibilidade do modelo a eles). Para cada uma, o valor é alterado, dentro de valores reais de sua amplitude, e se roda o modelo (simulações) mantendo-se constantes as demais condições. Uma comparação entre mudanças nos valores dos parâmetros e os resultados, fornece uma indicação da importância relativa da variável.

Outra maneira de se avaliar os modelos é através de sua precisão, definida em termos de três estágios de sua implementação: (i) verificação, calibração e (iii) validação.

Verificação é o processo pelo qual a lógica de programação é comparada com as intenções do modelador, ou seja, é verificado se o programa executa precisamente o que o modelador propôs.

Calibração refere-se aos ajustes dentro de uma amplitude conhecida - feitos para os parâmetros do modelo para dar a comparação 'mais precisa' entre os resultados simulados e os resultados observados a campo. Diz-se que o modelo é mais 'preciso' quando a simulação se aproxima mais dos dados observados. O uso indiscriminado da calibração torna o modelo de difícil extrapolação e pode descaracterizar parte do seu mecanismo. Além do mais, a calibração excessiva pode ocultar falhas que seriam importantes se conhecer.

O estágio de 'validação' está relacionado com o estabelecimento da similaridade das respostas do modelo, pela comparação das mesmas com um conjunto de observações independentes. Esta etapa muitas vezes não é possível pela complexidade da modelagem.

Segundo Costa (1997), a avaliação de modelos deve passar - e normalmente passa por duas avaliações distintas: uma subjetiva, que considera critérios como utilidade, simplicidade, elegância, e economia, principalmente; e outra objetiva, que utiliza critérios pré-definidos para se avaliar o modelo. É comum se observar na literatura científica, como parte do critério objetivo, o termo 'validação do modelo'. Nesse sentido é importante ressaltar que pelas definições apresentadas e discutidas acima, o modelo é uma teoria científica sobre o funcionamento de um certo sistema. Dessa forma, há que se considerar que uma teoria científica somente pode ser falsificada, nunca validada. Assim sendo, não é recomendável utilizar o termo validação, e passar a utilizar o termo correto para tais avaliações objetivas: teste do modelo.

Outro método bastante usado para a avaliação dos modelos é a comparação dos dados reais com os dados simulados. No entanto, é necessário ressaltar que tal teste é 
somente parte da avaliação total do modelo, e quando necessária sua utilização, deve ser baseada na prudência, evitando erros de interpretação. Ainda em relação aos dados observados e simulados, um dos métodos mais utilizados recentemente é o da análise de suas diferenças. Tal procedimento apresenta uma série de vantagens em relação ao convencionalmente utilizado, ou seja, a simples comparação de dados reais com dados simulados. Para que tal procedimento tenha sentido são necessárias algumas considerações, tais como, quais variáveis serão testadas e quais os níveis de precisão aceitáveis para essas variáveis (Costa, 1997).

Outro aspecto importante a ser considerado nos testes dos modelos é que tais testes devem ser feitos para longos períodos de tempo e nas mais diversas condições ambientais.

\section{Vantagens e limitações dos modelos}

As vantagens da utilização de modelos matemáticos, segundo Pessoa et al. (1997), implicam em um crescente aumento no uso, proposição e validação dos modelos, sendo elas: baixo custo, velocidade, completa informação, criação de cenários e proposição de cenários ideais.

A instalação, condução, avaliação e análise dos resultados exigem infra-estrutura e mão-de-obra especializada, que fazem com que a investigação experimental seja, muitas vezes, maior que a execução de um programa computacional. É importante lembrar que a aquisição de computadores está se tornando mais acessível a cada dia, mesmo em classes sociais que antes não tinham acesso a essa ferramenta. Durante a vida útil do equipamento, inúmeras simulações podem ser executadas, ao passo que no campo, em uma área determinada, só é possível executar um experimento por vez.

À medida que o problema adquire complexidade e aumenta de proporções, a utilização da modelagem adquire maior importância. Apesar de possuir um custo maior, a condução de experimentos não pode ser descartada. A modelagem deve auxiliar na decisão de quais os rumos a experimentação a campo deve seguir, como por exemplo, fazendo com que os recursos sejam melhores alocados e, assim, a qualidade das informações sejam mais relevantes à pesquisa.

Quanto à velocidade, a execução de um experimento que avalie, por exemplo, a absorção de uma quantidade de nitrogênio pelas raízes e a sua influência sobre a produtividade pode levar até meses para que seja completado. Porém, a simulação desse processo por intermédio de parâmetros de entrada e equações pode levar poucos segundos. Em oito horas de trabalho em um campo experimental pode-se fazer a aplicação de nitrogênio e capina, por exemplo, enquanto que com este mesmo tempo, é possível simular diversas condições e obter os mais diversos resultados.

Com a execução de um experimento, o número de informações é relativamente baixo, pois se restringe ao número de parâmetros avaliados. No entanto, com a simulação, tem-se os valores dos parâmetros de entrada e cada um dos valores calculados no decorrer do processo, até a obtenção do resultado final. Com a obtenção desse relatório é possível, então, visualizar todo o sistema, evidenciando pontos conflitantes e importantes na condução de novas investigações experimentais.

A simulação de condições diversas já é bem conhecida. Com ela, existe a possibilidade de criar cenários que ainda não são conhecidos, ou seja, cenários alternativos - muitos deles ainda não explorados em experimentos reais. A construção de condições ideais também é possível, o que em condições experimentais seria muito difícil de acontecer, mesmos que os experimentos sejam cuidadosamente executados. 
No entanto, é evidente que toda técnica - além das vantagens - apresente suas limitações. Uma boa aplicação de qualquer técnica exige que, além do domínio desta, haja pleno conhecimento de suas limitações.

Uma das limitações é a validação dos modelos e simuladores já existentes. Esse processo, às vezes, é demorado e requer conhecimento amplo do problema, além da obtenção dos dados em campo. Entretanto, sem a execução dessa etapa, todo resultado obtido por simulação poderá ser seriamente comprometido, repercutindo na obtenção de tendências equivocadas (Pessoa et al., 1997).

Quando o problema em estudo possuir mais de uma solução, fica difícil determinar qual delas seria a mais conveniente. Outro aspecto é com relação à confiabilidade e exatidão do modelo empregado. Somente por intermédio de uma seleção das bases de dados usadas para o desenvolvimento do modelo, ou em sua aplicação posterior, pode-se minimizar o problema (Dourado Neto et al., 1998b). De que forma os dados referentes às variáveis foram coletadas? Qual a confiabilidade desses dados? Um modelo é tão preciso e possui confiabilidade de acordo com a base de dados que utiliza em seu desenvolvimento influenciando diretamente a simulação.

Boote et al. (1996) citaram algumas limitações para a utilização da modelagem, como o custo de obtenção dos dados (por exemplo, fotos de satélites, dados provenientes de instrumentação especializada), a variabilidade espacial (em aplicações na experimentação de campo, a variabilidade do solo é muito grande de um local para o outro, causando diferentes produtividades de grãos e esta área é tratada como única), a técnica para o entendimento de alguns dados de entrada, a variabilidade temporal e a qualidade dos dados (deficiência na calibração dos sensores).

A eficiência do processo de modelagem depende do conhecimento do modelador que vai desenvolver o modelo.
As áreas que dão suporte a modelagem vão depender, sobre tudo, de qual o objetivo da modelagem. Algumas áreas do conhecimento na modelagem em agricultura são fundamentais, como por exemplo, agricultura, fisiologia, matemática, estatística, meteorologia, manejo cultural, ecologia, climatologia e lógica de programação, dentre outras. Dessa forma, existe a necessidade de equipes 'multidisciplinares' para o desenvolvimento dos modelos e, com isso, a ampliação de sua utilização.

Costa (1997) ressaltou que o desenvolvimento e a aplicação de modelos de simulação, em conjunto com a experimentação convencional em maior escala no Brasil, é um desafio que se deve seguir e que, sem dúvida, tornará as atividades científicas mais eficientes.

\section{CONCLUSÕES}

O desenvolvimento e a aplicação da modelagem em agricultura é uma valiosa ferramenta que pode - e deve - servir para a orientação de pesquisas, gestão de tecnologia e até decisões políticas. Seu uso no Brasil, apesar de ainda incipiente, tem aumentado nos últimos anos, muito embora em função do maior acesso de usuários a computadores e programas computacionais, mas também devido à melhoria de técnicas de modelagem e da maior capacidade dos computadores na realização de cálculos.

É importante que o meio acadêmico faça bom uso desta ferramenta, seja na condução de estudos hipotéticos, avaliando e comparando os resultados obtidos com suas próprias experiências, mas também na difusão deste conhecimento em sala de aula, na formação de massa crítica, e em eventos voltados à difusão da informação.

O conhecimento 'interdisciplinar' possibilita a realização de simulações mais acuradas da dinâmica do crescimento de 
culturas e de sistemas agrícolas, mas para que de fato haja esta agregação de conhecimento, muitos modelos devem ser simplificados em submodelos. Esta simplificação de modelos é importante para a melhoria no entendimento dos processos e de suas respostas, mas deve-se estar atento às suas limitações.

Ressalta-se, aqui, que a proposta do uso de modelos não substitui a experimentação convencional, nem tampouco se deseja obter, através do modelo, uma única e exclusiva resposta para questões de natureza complexa e mutável. O que se pretende sim é tornar a atividade científica mais eficiente e, por conseguinte, fazer com que os resultados e descobertas oriundos desses trabalhos estejam disponíveis aos segmentos de interesse, de maneira mais ágil e menos onerosa.

\section{REFERÊNCIAS}

ADDISCOTT, T.M. Simulation modelling and soil behaviour. Geoderma, Amsterdam, v.69, p.15-40, 1993.

ANDRADE, F.H.; UHART, S.; ARGUISSAIN, G. Produtividade potencial de maiz em alcarce: analisis de los fatores que los determinan. Balcarce: Instituto Nacional de Tecnologia Agropecuária, 1991. 11 p. (Boletim Técnico, 101).

BOOTE, K.J.; JONES, J.W.; PICKERING, N.B. Potential uses and limitations of crop models. Agronomy Journal, Amsterdam, v.88, p.704-716, 1996.

BOUMAN, B.A.M.; VAN KEULEN, H.; VAN LAAR, H.H.; RABBINGE, R. The 'School of de Wit' crop growth simulation models: a pedigree and historical overview. Agricultural Systems, Amsterdam, v. 52, p.171198, 1996.

CORRÊEA, S.T.R. Adaptação do modelo LINTUL (Light Interception and
Utilization) para estimação da produtividade potencial da cultura de soja. 2008. 106p. Dissertação (Mestrado em Fitotecnia) - Escola Superior de Agricultura "Luiz de Queiroz", Universidade de São Paulo, Piracicaba, 2008.

COSTA, L.C. Modelagem e simulação em agrometeorologia. In: CONGRESSO BRASILEIRO DE AGROMETEORO LOGIA. 1997. Piracicaba. Anais...

Piracicaba: SBA, 1997. p.3-6

DOURADO NETO, D.; TERUEL, D.A.; REICHARDT, K.; NIELSEN, D.R., FRIZZONE, J.A.; BACCHI, O.O.S. Principles of crop modeling and simulation: I. Uses of mathematical models in agricultural science. Scientia Agricola, v.55, p.46- 50, 1998a.

DOURADO NETO, D.; TERUEL, D.A.; REICHARDT, K.; NIELSEN, D.R., FRIZZONE, J.A.; BACCHI, O.O.S Principles of crop modeling and simulation: II. The implications of the objective in model development. Scientia Agricola, v.55, p.51-57, 1998b.

DOURADO NETO, D. Modelos fitotécnicos referentes à cultura do milho. 1999. 229p. Tese (LivreDocência em Fitotecnia) - Escola Superior de Agricultura "Luiz de Queiroz", Universidade de São Paulo, Piracicaba, 1999.

DOORENBOS, J.; KASSAM, A.H. Efeito da água no rendimento das culturas. Tradução de HEYI, H. AA DE SOUZA, F.A.V DAMASCO, JF DE MEDEIROS. Campina Grande: UFPB, 1994. 306p. (Estudos FAO: Irrigação e Drenagem, 33).

DUNCAN, W.G. Maize. In: EVANS, L.T., ed. Crop physiology: some Case histories. London, Cambridge University Press, 1975. cap.2, p.23-50. 
ITTERSUM, M.K. VAN; LEFFELAAR, P.A.; KEULEN, H. van; KROPFF, M.J.; BASTIAANS, L.; GOUDRIAAN, J. On approaches and applications of the Wageningen crop models. European Journal of Agronomy, Amsterdam, v. 18, p. 201234, 2003.

JONES, J. W.; BOOTE, K. J.; JAGTAP, S. S.; WILKERSON, G. G.; HOOGENBOOM, G.; MISHOE, J. W. SOYGRO V5.4-Technical documentation. Gainesville, FL: Agricultural Engineering Department and Agronomy Department University of Florida, 1987. 170p.

KEULEN, H. VAN.; PENNING DE VRIES, F.W.T.; DRESS, E.M. A summary model for crop growth. In: PENNING DE VRIES, F.W.T.; VAN LAAR, H. H. (Ed). Simulation of plant growth and crop production. Wageningem: Pudoc, 1982. p.87-97.

KEULEN, H.VAN.; WOLF, J. Modeling of agricultural production: Weather, soils and crops. Wageningen: Pudoc, 1986. 463p.

KROPFF, M.J.; MATTEWS, R.B.; VAN LAAR, H.H.; BERGE, H.F.M. The rice model Oryzal and its testing. In: MATTHEWS, R.B.; KRPFF, M.J.; BACHELET, D.; VAN LAAR, H.H. (Ed.) Modeling the impact of climate change on rice production in Asia. Manila: International Rice Research Institute, 1995. p.27-50.

LAW, A.M.; KELTON, W.D. Simulation modeling and analysis. 2 ed. New York: MacGraw-Hill, 1991.

LORENÇONI, R.; DOURADO-NETO, D.; HEINEMANN, A.B.; SILVA, A.N. da; SILVA, L.C.S. Calibração do modelo ORYZA-APSIM para o arroz de terras altas no Brasil. Revista de Agricultura, Piracicaba, v.85, n.3, p.237-244, 2010.
MARIN, F. R.; SENTELHAS, P. C.; UNGARO, M. R. G. Perda de rendimento potencial da cultura do girassol por deficiência hídrica, no Estado de São Paulo. Scientia Agrícola, Piracicaba, v.57, n.1, p. 1-6, 2000.

McCREE, K. J. An equation for the rate of respiration of white clover plants growth under controlled conditions. In: SETLIK, I., (Ed.). Prediction and measurement of photosynthetic productivity. Wageningen, Pudoc: Elsevier, 1970. p.221-229

MEINKE, H., HOCHMAN, Z. Using seasonal climate forecasts to manage dryland crops in northern Australia. In: HAMMER, G.L.; NICHOLLS, N.; MITCHELL, C. (Ed.). Application of seasonal climate forecasting in agricultural and natural ecosystems: the Australian experience. Amsterdam: Kluwer Academic, 2000. p. 149-165.

MUNAKATA, K. Simulation of population photosynthesis and dry matter production. In: MATSUO, T.; KUMAZAWA， K.; ISHII, R.; ISHIHARA, K.; HIRATA, H. (Ed.) Science of the rice plant. Tokyo, Physiology, Food and Agricultural Policy Research Center, 1995. v.2, p.671-684.

PEREIRA, A.R.; ANGELOCCI, L.R.; SENTELHAS, P.C. Agrometeorologia: fundamentos e aplicações práticas. Guaíba: Agropecuária, 2002. 478p.

PAUTIAN, K.; PARTON, W.J.; PERSSON, J. Modeling soil organic matter in organic-amended and nitrogen fertilized long-term plots. Soil Science Society of America Journal, v.56, p.476-488, 1992.

PEDRO JÚNIOR, M. J.; CAMARGO, M. B. P.; BRUNINI, O.; ALFONSI, R. R.; 
ORTOLANI, A. A.; MIRANDA, M. A. C. Teste de modelo agrometeorológico para estimativa da produtividade potencial da cultura da soja de ciclo precoce. In: CONGRESSO BRASILEIRO DE AGROMETEOROLOGIA， 3， 1983, Campinas. Anais... Campinas, 1983, p. 11-17.

PENNING de VRIES, F.W.T. System analysis and models of crop growth. In: PENNING DE VRIES, F.W.T.; VAN LAAR, H.H. (Ed.). Simulation of plant growth and crop production. Pudoc, Wageningen: Elsevier, 1982. p.9-22.

PEREIRA, A.R.; MACHADO, E.C. Análise quantitativa do crescimento de comunidades Vegetais. Campinas: Instituto Agronômico, 1987. 33p.

PESSOA, M.C.P.Y.; LUCHIARI JUNIOR, A.; FERNANDES, E.N.; LIMA, M.A. Principais modelos matemáticos e simuladores utilizados para a análise de impactos ambientais das atividades agrícolas. Brasília, EMBRAPA, 1997, 44p.

SANTOS, J.E. dos Procedimentos básicos para a modelagem ecológica. Ciência e Cultura, v.36, n.6, p.983-992, 1986.

SPITTERS, C.J.T.; TOUSSAINT, H.A.J.M.; GOUDRIAAN, J. Separating the diffuse and direct component of global radiation and its implications for modeling canopy photosynthesis Part I. Components of incoming radiation. Agricultural and Forest Meteorology, v. 38, p.217-229, 1986.

THORNLEY, J. H. M. Respiration, growth and maintenace in plants. Nature, v.227, p.304-305, 1970.

THORNLEY, J.H.M. Mathematical models in plant physiology: a quantitative approach to problems in plant and crop physiology, London: Academic Press., 1976. 318p.

THORNLEY, J.H.M.; JOHNSON, I.R. Plant and crop modeling. Oxford: Oxford University Press, 1990. 669p.

WIT, C.T. de; GOUDRIAAN, J. Simulation of ecological processes. Wageningen, The Netherlands: Centre for Agricultural Publishing and Documentation, 1974. 159p.

WIT, C.T. de Simulation of assimilation, respiration and transpiration of crops. Wageningen, The Netherlands: Centre for Agricultural Publishing and Documentation, 1978. 141p.

WIT, C.T.; GOUDRIAAN, A.H. Simulation of ecological processes. 2 nd ed. New York: John Wiley, 1978. 140 p.

ZADOKS, J.C.; RABBINGE, R. Modelling to a purpose. In: C.A. Gilligan, Editor, Advances in Plant Pathology, Vol. 3, Mathematical Modelling of Crop Disease, Academic Press, London, pp. 231-244, 1985. 